AL-AZHAR UNIVERSITY

BULLETIN OF THE FACULTY OF

LANGUAGES \& TRANSLATION

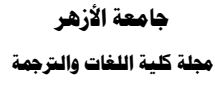

\title{
Der Lernprozess aus neurodidaktischer Perspektive
}

Doaa Abd Al-Hay Azab

Al Al-Azhar Universität

Sprachen- und Übersetzungsfakultät

Abteilung für Germanistik 


\section{Learning from the Perspective of Educational Neuroscience}

Doaa Abdel Hay Azab

German Language Department, Faculty of Languages \& Translation, Al Azhar University, Cairo, Egypt.

Email: doaa.azab.2010@azhar.edu.eg

\section{ABSTRACT:}

Learning is a life-long process which does not only happen in classrooms. In fact, once one stops learning, one starts dying. The process of learning aims at self-development. Hence, this process became a research field for many sciences including the emerging scientific field, 'educational neuroscience'. In the late 1980s, Gerhard Preiss proposed this new discipline to integrate recent brain researches for enhancing the educational process. Educational neuroscience is considered as a bridge between neurology and pedagogy, it also has a strong relation to educational psychology. Thus, educational neuroscience is an integration of these three disciplines to boost the educational process and achieve more effective learning.

Keywords: Learning, Perspective, Education, Educational Neurology, Pedagogy.

$$
\text { قدعاء عبد الجي عزب التعلم من منظور علم الأعصباب التعليمي }
$$

التعلم هو عملية تتم طوال حياة الأنسان فهي ليست امر خاص بالطالب وداخل الفصل الدراسي فقط. فعندما يتوقف المرء عن التعلم يصبح حينها ميت بالفعل. فعملية التعلم هي عملية تطور للذات ومن اجل ذلك أصبحت عملية التعلم مجال بحث في كثير من العلوم ومن ضمن هذه العلوم الحديثة علم الأعصاب التعليمي (Neurolodidactic). تم ادراج مصطلح علم الأعصاب التعليمي في نهاية عام 1980 من قبل العالم Gerhard Preiß من اجل دمج ابحاث المخ الحديثة في تطوير العملية التعليمية. فيعد علم الأعصاب التعليمي جسر بين علم الأعصاب وعلم التربية كما ان له علاقة قوية بعلم النفس التعليمي. فيعتبر دمج لهذه العلوم الثلاث بهدف تطوير العملية التعليمية والوصول الي التعلم الفعال.

$$
\text { الكلمات المفتاحية: التعلم ، منظور ، التعليم ، علم الأعصاب التعليمي ، علم التربية. }
$$




\section{Einleitung}

Lernen ist ein lebenslanger Prozess. Es ist kein Thema für Studenten innerhalb der Klassenräume. Wenn man nicht mehr lernen will, stirbt man. Das Lernen ist für uns ein dauerhafter Entwicklungsprozess, deshalb ist es ein wichtiges Forschungsfeld in vielen Wissenschaften. $\mathrm{Zu}$ den modernen Wissenschaften, die sich mit dem Lernprozess beschäftigen, gehört die Neurodidaktik.

Der Terminus „Neurodidaktik“ wurde Ende der 1980er Jahre vom Fachdidaktiker Gerhard Preiß eingeführt, um Ergebnisse der Hirnforschungen in die Didaktik miteinzubeziehen. ${ }^{1}$

Neurodidaktik wird oft als Brückendisziplin zwischen der Neurowissenschaft und der Pädagogik definiert. Insbesondere die didaktische Psychologie spielt hier eine wichtige Rolle.

In diesem Wissenschaftsfeld werden Neurowissenschaften, Psychologie und Pädagogik mit dem Ziel, den Lehr- und Lernprozess zu optimieren, zusammengeführt.

In dieser Forschung wird der Lernprozess aus dem Blickwinkel der Neurodidaktik betrachtet.

\subsection{Problemstellung}

Viele Menschen sowohl der Lehrende als auch der Lernende betrachten das Lernen als die Lieferung von Informationen und das Pauken von Fakten. Sie unterscheiden nicht zwischen dem Auswendiglernen und dem sinnvollen Lernen. Darüber hinaus kennen sie nicht die entscheidende Rolle der Emotionen beim Lernen.

Aus diesen Problematiken heraus entstand das Interesse der vorliegenden Forschung, in der ich mich mit den folgenden Fragen beschäftige:

- Was ist Lernprozess?

- Inwieweit beeinflusst die Emotionen den Lernprozess?

- Was ist das effektivste Lernen aus neurodidaktischer Sicht?

1 Vgl. Friedrich, Gerhard: Allgemeine Didaktik und Neurodidaktik, Peter lang Verlag, Frankfurt am Main 2005, S. 8. 


\subsection{Ziel der Arbeit}

Die vorliegende Forschung macht es sich zum Ziel, den Lernprozess aus der Sicht der modernen Hirnforschungen zu analysieren und zu unterstützen.

Diese Arbeit sieht sich als ein Beitrag zur Berücksichtigung der Rolle der Emotionen beim Lernen und zur Erkennung der Lehr-Lern-Prinzipien der Neurodidaktik.

\subsection{Methode der Arbeit}

In dieser Forschung wird die deskriptiven und analytischen Forschungsmethoden angewandt. Hierbei wird der Lernprozess aus neurowissenschaftlicher Perspektive betrachtet. 


\section{Der Begriff „Lernen“}

Jede Sekunde begegnet das menschliche Gehirn einer Flut von Informationen, die es nicht passiv speichert. Diese Informationen werden in spezifischen synaptischen Netzwerken verarbeitet, analysiert, bewertet sowie schließlich im Langzeitgedächtnis $^{2}$ abgespeichert. Aus diesen Vorgängen ergibt sich eine Veränderung der Strukturen neuronaler Netzwerken bzw. Verbindungen, die als Synapsen bezeichnet werden. Wenn man etwas gelernt hat, heißt es, dass ein Aufbau oder Abbau innerhalb neuronaler Verbindungen bzw. Synapsen passiert ist. Dementsprechend wird Lernen wie folgt definiert:

„Lernen ist aus neurobiologischer Sicht die Veränderung der Plastizität neuronaler Strukturen in unserem Gehirn, die durch den Auf- und Abbau von Neuronen sowie die Verstärkung bzw. Abschwächung neuronaler Verschachtelungen bedingt wird. " ${ }^{\text {3 }}$

Folglich kann man sagen, dass jeder Lernvorgang ein Umstrukturierungsprozess vorheriger aufgenommener Wissensbestände im Gehirn ist.

Der Neurobiologe Manfred Spitzer betrachtet das Lernen folgendermaßen:

„Modifikation synaptischer Übertragungsstärke. \{...\} Je aktiver ein neuronales Gewebe in einem bestimmten Bereich der Gehirnrinde ist, desto eher findet in ihm Veränderung von Synapsenstärke und damit Lernen statt. "4

Dies bedeutet, dass je stärker Synapsen miteinander verbunden sind, desto leichter das Gelernte später abgerufen werden kann.

Neurowissenschaftler betrachten den Lernprozess als einen hochkomplexen kognitiven Vorgang, an dem alle menschliche Organe und Dispositionen beteiligt sind. ${ }^{5}$ In anderen Worten: Körper und Gehirn, sowie auch Emotionen bilden beim Lernen eine dynamische Einheit; somit ist alles miteinander verknüpft.

Gerhard Roth hat bei seiner Erklärung zum Lernen die beobachtbare Verhaltensveränderung berüchsichtigt. In seinem Buch „Bildung braucht Persönlichkeit" hat er Lernen wie folgt definiert:

\footnotetext{
${ }^{2}$ Langzeitgedächtnis ist eine Gedächtnisform, die unbegrenzte Speicherkapazität und Behaltensdauer besitzt. Dort werden die Informationen langfristig abgespeichert und bei Bedarf abgeruft.

3 Brünner, Ines: Gehirngerechtes Lernen mit digitalen Medien. Ein Unterrichtskonzept für den integrativen DaF-Unterricht, TU Berlin, Berlin 2008, S. 28.

${ }^{4}$ Spitzer, Manfred: Lernen. Gehirnforschung und die Schule des Lebens, Spektrum Akad. Verlag, Heidelberg 2003, S. 146.

5 Vgl. Ulrich, Herrmann: Neurodidaktik. Grundlagen und Vorschläge für gehirngerechtes Lehren und Lernen, Beltz Verlag, Weinheim und Basel 2006, S. 182.
} 
„Lernen ist eine universell verarbeitete Fähigkeit zur mittel- und langfristigen Anpassung eines Organismus an seine Umwelt. "6

Im Laufe der Zeit haben die Wissenschaftler versucht, den Lernprozess zu erfassen und exakt zu beschreiben. Zu diesem Zweck haben sie verschiedene lerntheoretische Ansätze entworfen. Im folgenden Ablauf wird eine Übersicht über die wichtigsten Lerntheorien gegeben.

\section{Lernen aus lerntheoretischer Sicht}

Es gibt eine Vielzahl von Lerntheorien, die den Lernvorgang beschreiben. Diese Lerntheorien ermöglichen es, die verschiedenen Lernformen oder -arten zu verstehen und dadurch den Lernunterricht zu optimieren.

In dieser Arbeit werden drei bedeutende und weit verbreitende Lerntheorien herauskristallisiert. Diese sind der Behaviorismus, der Kognitivismus und der Konstruktivismus.

\subsection{Der Behaviorismus}

Der Terminus „Behaviorismus” leitet sich aus dem englischen Wort „behavior“ ab, auf Deutsch „Verhalten“. Diese Lernichtung wurde in der ersten Hälfte des 20. Jahrhunderts von John B. Watson und Edward Thorndike erfunden und in den 1950er Jahren von Burrhus Frederic Skinner weiterentwickelt. ${ }^{7}$

Aus behaviorischer Sicht wird das Lernen als äußerlich beobachtbare Verhaltensveränderung interpretiert. ${ }^{8}$ Das heißt, dass interne Komponenten wie Motive und informationsverarbeitende Vorgänge in dieser Interpretation nicht berücksichtigt werden.

Edward L. Thorndike, einer der berühmtesten Behavioristen, beschreibt den Lernprozess als Assoziation zwischen einem Reiz und einer Reaktion. Deshalb werden behavioristische Theorien als $\mathrm{S}$-R-Theorien ( $\mathrm{S}=$ stimulus, $\mathrm{R}=$ response) bezeichnet. Der Lernende nimmt Reize auf, die in seinemVerhalten zur Veränderung führen. Diese Veränderungen werden als „Reaktionen” bezeichnet. Wie die Reize im Gehirn verarbeitet werden, ist nicht von Bedeutung. Das Gehirn wird dementsprechend als eine Art Black Box betrachtet. ${ }^{9}$

\footnotetext{
${ }^{6}$ Roth, Gerhard: Bildung braucht Persönlichkeit. Wie Lernen gelingt, Klett-Gotta Verlag, Stuttgart 2011, S.92.

${ }^{7}$ Vgl. Brünner, Ines: 2008, S.46.

8 Vgl. Auer, Christina: Fremdsprachenerwerb. Erwachsener in der Weiterbildung. Entwicklung eines teilnehmerorientierten Unterrichtkonzepts, W. Bertelsmann Verlag, Bielefeld 2013, S. 38.

${ }^{9}$ Vgl. Brünner, Ines: 2008, S.46.
} 
Die Behavioristen haben verschiedene Experimente durchgeführt, um ihren Lernansatz zu unterstützen. Zu diesen Experimenten gehört die Untersuchung von Iwan Pawlow.

Pawlow führte sein Experiment mit Hunden durch. Dabei ließ er den Hunden Essen bringen. Die Hunde produzierten beim Geruch des Essens Speichel. Später wurde das Erklingen eines Glöckchens vor der Essenausgabe hinzugefügt. Dieser Vorgang wurde mehrmals wiederholt. Nach einiger Zeit begannen die Hunde bereits beim Erklingen der Glocke Speichel zu produzieren. Sie hatten also gelernt, dass beim Erläuten der Glocke das Essen folgte. John B. Watson führte ein ähnliches Experiment, mit einem neun Monate alten Jungen namens Albert durch. Albert wurde eine weiße Ratte präsentiert. Er zeigte beim ersten Mal keine Angst vor dem Tier. Später wurde Albert die weiße Ratte in Verbindung mit einem lauten Knall präsentiert, woraufhin Albert erschrak und anfing, zu weinen. Dies wurde mehrmals wiederholt. Mit der Zeit fing Albert beim Anblick der Ratte stets an zu weinen, auch ohne den lauten Knall zu hören. ${ }^{10}$

Es geht also um Input und Output. Der Lernende wird passiv betrachtet: Seine Rolle beim Lernprozess beschränkt sich lediglich auf die Aufnahme von Informationen in Form von Reizen und die Reaktionen auf diese Reize. Der Lehrer repräsentiert dabei den Vermittler von Fachwissen. In anderen Worten hat der Lehrer die Kontrolle über den ganzen Lernprozess. Darüber hinaus basiert das Lernen auf der Wiederholung und Imitation von Wissen. Dieses Verständnis des Lernprozesses passt sehr gut mit dem traditionellen Frontalunterricht zusammen.

\subsection{Der Kognitivismus}

Der Kognitivismus entwickelte sich in der zweiten Hälfte des 20. Jahrhunderts aus dem Behaviorismus. $\mathrm{Zu}$ den berühmtesten Angehörigen dieser Richtungen gehören George Miller und Jerome Bruner ${ }^{11}$

Anders als der Behaviorismus berücksichtigt diese Richtung die kognitiven und mentalen Prozesse, die sich im Gehirn vollziehen, sowie die innerpsychischen Vorgänge. Zu den kognitiven Prozessen gehören z.B. Denken, Sicherinnern, Wahrnehmung, Motivation und Vorstellung. Das heißt, dass der Kognitivismus das menschliche Gehirn nicht als Black Box betrachtet, sondern als ein wichtiges System zur Informationsaufnahme und -verarbeitung. In diesem Zusammenhang hat Christina Auer folgendes gesagt:

\footnotetext{
${ }^{10} \mathrm{Vgl}$. https://blog.cognifit.com/de/lerntheorien/, am 4.1.2019.

${ }^{11} \mathrm{Vgl}$. http://lexikon.stangl.eu/187/kognitivismus/, am 6.1.2019.
} 
„Der Kognitinismus interpretiert Lernen nicht nur mehr als beobachtbare Verhaltensveränderung, determiniert durch die Umwelt, Lernen wird zu einem internen Vorgang, bei dem die mentalen Verarbeitungsprozesse des Lernenden im Zentrum stehen. Demnach wird auch der Vorerfahrung eine bedeutende Rolle zugesprochen. \{...\}Kognitive Prozesse führen vor allem durch Entdecken, Wiederentdecken oder Wiedererkennen zum Wissenserwerb und umfassen visuelle, auditive Bereiche gleichermaßen. "12

Man geht davon aus, dass der Lernprozess auf Hypothesenbildung, Informationensanalyse, selbständige Informationserschließung und -speicherung beruht. Darüber hinaus spielen das deklarative sowie das produrale Gedächtnis beim Aufbau vom neuen Wissen eine entscheidene Rolle.

Aus kognitiver Perspektive wird das Lernen mit den Ausdrücken ,entdeckendes Lernen” und „sinnvolles Lernen” beschrieben. Das heißt, dass der Lernende nicht passiv Informationen rezipiert, sondern sich aktiv am Lernprozess beteiligt. Diese Lernrichtung verändert die stereotypische Rolle des Lehrers vom Wissensvermittler zum Lernbegleiter und -berater.

\subsection{Der Konstruktivismus}

Diese Lernrichtung ist die Entwicklungsform des Kognitivismus. Konstruktivismus sieht das Lernen als einen individuellen mentalen Prozess der Wissenskonstruktion, den der Lernende selbständig steuert. Der Lernende eignet sich nicht an, was vermittelt wird, sondern, was er gemäß seiner Erfahrungen, Vorstellungen, Gesellschaft will und selbst entscheidet. D.h., dass der Lernprozess stark vom Lernenden abhängt. Seine Erfahrungen, Vorstellungen sowie sein Umfeld, seine gesellschaftliche Zugehörigkeit und Emotionen kommen dabei ins Spiel. Dies wird von der folgenden Aussage bestätgt:

Aufgrund dessen spielt das Vorwissen des Lernenden eine wichtige Rolle beim Aufbau neues Wissens, da die neuen Informationen, bevor sie im Gehirn gespeichert werden, gefiltert und mit dem vorhandenen Wissen verknüpft werden.

$\mathrm{Zu}$ den Vertretern dieser Lernrichtung zählen Jean Piagets, George A. Kelly, Ernst von Glasersfeld und Heinz von Förster. ${ }^{13}$

Im Sinne des Konstruktivismus steht der Lernende als Konstrukteur des Wissens im Mittelpunkt des Lernprozesses. Der Lehrende wird, wie im Kognitivismus, als Lernbegleiter und Lernberater angesehen, der autonomes Lernen fördern soll.

\footnotetext{
${ }^{12}$ Auer, Christina: 2013, S. 41.

${ }^{13} \mathrm{Vgl}$. https://user.phil-fak.uni-duesseldorf.de/ petersen/WiSe1516 InfowiColl/Diskurs Konstruktivismus.pdf, am 6.1.2019.
} 
Zur Zusammenfassung der drei erläuterten Lerntheorien dient folgende Tabelle:

\begin{tabular}{|c|c|c|c|}
\hline & Behaviorismus & Kognitivismus & Konstruktivismus \\
\hline Gehirn & Black Box & $\begin{array}{l}\text { Informationsverarbeit- } \\
\text { ungssystem }\end{array}$ & $\begin{array}{l}\text { selbstreferentielles } \\
\text { zirkuläres System }\end{array}$ \\
\hline Lernen & $\begin{array}{lr}\text { Lernen } & \text { durch } \\
\text { Input- } & \text { output } \\
\text { Relation } & \end{array}$ & $\begin{array}{l}\text { Lernen durch Einsich } \\
\text { und Erkenntnis }\end{array}$ & $\begin{array}{l}\text { Lernen durch } \\
\text { persönliche } \\
\text { Erfahrungen und } \\
\text { Interpretationen. }\end{array}$ \\
\hline Lernerfolg & $\begin{array}{l}\text { Beobachtbares } \\
\text { Verhalten }\end{array}$ & $\begin{array}{l}\text { Verarbeitung } \\
\text { Informationen }\end{array}$ & $\begin{array}{l}\text { Konstruktion des } \\
\text { Wissens }\end{array}$ \\
\hline Wissen & Faktenwissen & Prozeduren, Verfahren & soziale Praktiken \\
\hline Gedächtnisformen & deklarativ & deklarativ, prozedural & Emotional \\
\hline Lerner & $\begin{array}{l}\text { nimmt } \\
\text { Informationen auf }\end{array}$ & $\begin{array}{l}\text { verarbeitet } \\
\text { Informationen }\end{array}$ & konstruiert Wissen \\
\hline Lehrer & $\begin{array}{l}\text { Wissensvermittler, } \\
\text { Autorität }\end{array}$ & Tutor, Berater & Moderator \\
\hline Implizites Lehrziel & $\begin{array}{l}\text { Erinnern, Merken, } \\
\text { Wiedererkennen }\end{array}$ & $\begin{array}{l}\text { Probleme } \\
\text { Wissen nutzen }\end{array}$ & $\begin{array}{l}\text { Situationen } \\
\text { bewältigen, } \\
\text { reflektierend } \\
\text { handeln }\end{array}$ \\
\hline $\begin{array}{l}\text { Überprüfung des } \\
\text { Lehrerfolgs }\end{array}$ & $\begin{array}{l}\text { Reproduktion } \\
\text { korrekter } \\
\text { Antworten }\end{array}$ & $\begin{array}{ll}\text { Auswahl und } \\
\text { Anwendung } \\
\text { Methode }\end{array}$ & $\begin{array}{l}\text { Bewältigung } \\
\text { komplexer } \\
\text { Situationen }\end{array}$ \\
\hline
\end{tabular}

$(\text { Tabelle } 1)^{14}$

${ }^{14}$ Vgl. Brünner, Ines: 2008, S.52. 
Die folgende Abbildung verdeutlicht diese Theorien ebenfalls:
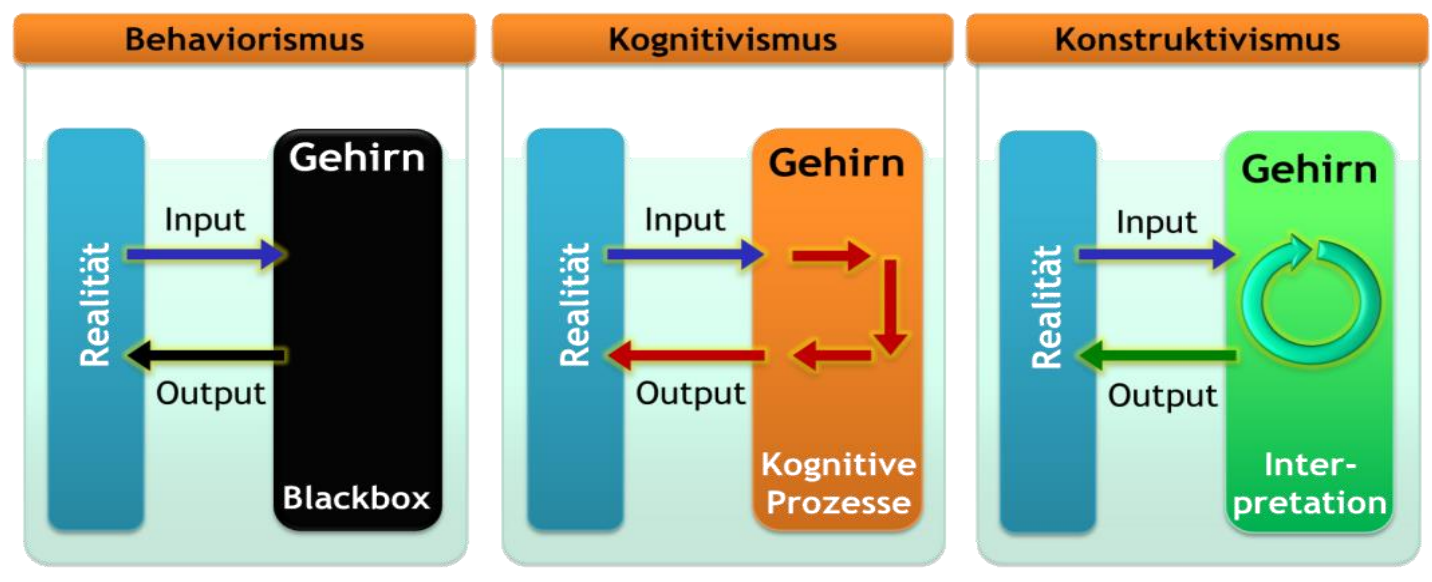

(Abbildung 1) ${ }^{15}$

Die vorausgehenden Theorien zeigen auf, dass das Lernen ein hochkomplexer Prozess ist, an dem Vorerfahrungen und Emotionen des Lernenden beteiligt sind. Deshalb wird die Rolle der Emotionen beim Lernen im folgenden Abschnitt näher thematisiert.

\section{Emotionen und Lernen}

In den letzten Jahren erschienen zahlreiche Studien, die die Relevanz der Emotionen beim Lernen hervorheben. Zahlreiche Befunde bestätigen, dass Emotionen die Intensität der Speicherung von Gedächtnisinhalten modulieren. Der Lernerfolg hängt also mit den Emotionen und dem emotionalen Zustand des Lernenden zusammen. $\mathrm{Zu}$ den wichtigsten Studien gehören die Untersuchungen des Neurobiologen Ralph Adolph, der sich unter anderem mit dem Einfluss von Emotionen auf kognitive Prozesse beschäftigt.

Im Jahre 1997 führte er eine Studie zur Wirkung der Amygdala ${ }^{16}$ auf die langfristige Speicherung vom Faktenwissen durch. Ihm standen dabei zwei Patienten mit beidseitigem Ausfall der Amygdala, bzw. einer Amygdalaläsion, zur Verfügung. Den beiden Patienten wurde eine Geschichte mit neutralen und auch mit emotional grausamen Sequenzen vorgelesen. Einen Tag später wurden sie einem Gedächtnistest mit detaillierten Fragen zum Inhalt der Geschichte unterzogen. Als Kontrollgruppen hat Adolph eine Gruppe von Patienten mit anderen Gehirnstörungen und eine weitere

\footnotetext{
15 Http://www.sciencetonic.de/200_dm_025_konzepte.html, am 6.1.2019.

16 Amygdala ist eine Ansammlung von Neuronen im Vorderhirn, die pradigmatisch für die Funktion von Emotionen stehen.
} 
Gruppe gesunder Versuchspersonen ausgewählt, denen ebenfalls die Geschichte präsentiert wurde und denselben Gedächtnistest machen mussten. Die beiden Kontrollgruppen sowie auch die Testgruppe mit der Amygdalaläsion haben den Gedächtnistest nicht nur am darauffolgenden Tag, sondern auch eine Woche später durchgeführt. Das Ergebnis des Gedächtnistestes hat einerseits gezeigt, dass die beiden Kontrollgruppen sich die erregenden Szenen detaillierter als die Szenzen mit neutralen Ergebnissen gemerkt hatten und wiedergeben konnten. Andererseits konnte bei der Testgruppe mit Amygdalaläsion kein Unterschied zwischen den emotionalen und neutralen Szenen festgestellt werden. Die Testpersonen konnten die emotionalen Szenen weniger gut als die beiden Kontrollgruppen wiedergeben. ${ }^{17}$

Diese Studie bestätigt die Annahme, dass die Amygdala eine Schlüsselrolle bei der Speicherung vom Wissen spielt.

Um eine weitere, ähnliche Studie aufzuführen, wird nun die Untersuchung der Psychologin Elizabeth A. Phelps beleuchtet.

Im Labor von Phelps wurden Patienten mit Amygdalaläsion und eine gesunde Kontrollgruppe gebeten, eine Liste von Tabuwörtern und neutralen Wörtern zu lesen. Eine Stunde später sollten sich alle Probanden an die Wörter erinnern. Während sich die Kontrollgruppe an neutrale Wörter kaum erinnern konnte und die Tabuwörter besser wiedergeben konnte, zeigten Amygdalapatienten einen Gedächtnisabfall sowohl für die neutralen als auch für die Tabuwörter. ${ }^{18}$

Um den Einfluss der Emotionen auf das Lernvermögen näher zu verstehen, wird im Folgenden ein Blick auf das emotionale System im menschlichen Gehirn, das limbische System, geworfen.

\subsection{Das limbische System}

Der Begriff „limbisches System“ stammt vom französischen Arzt Paul Broca, der dieses Gefühlssystem im Jahre 1878 entdeckt hat. Diese Bezeichnung leitet sich vom lateinischen Wort „limbus“ ab, das auf Deutsch „Saum“ bedeutet. Damals bezog sich diese Bezeichnung nur auf das ringförmige Areal, das den Hirnstamm umgibt.1949 hat der amerikanische Mediziner und Hirnforscher Paul Mclean das Konzept des limbischen Systems weiterentwickelt und andere Gehirnareale $\mathrm{zu}$ diesem hinzugefügt. Darüber hinaus hat er die Funktionen dieses Systems näher bestimmt. ${ }^{19}$

\footnotetext{
17 Vgl. Arnold, Margret: Aspekte einer modernen Neurodidaktik. Emotionen und Kognitionen im Lernprozess, Verlag Ernst Vögel, München 2002, S. 92-93.

18 Ebd., S.94.

${ }^{19} \mathrm{Vgl}$. https://www.dasgehirn.info/grundlagen/anatomie/das-limbische-system, am 1.7.2018.
} 
Der Terminus „limbisches System” bezieht sich also auf verschiedene Teile im menschlichen Gehirn, die vor allem für die Emotionen zuständig sind . Es ist der eigentliche Kontrolleur des Lernerfolgs, weil es den ganzen Lernprozess steuert und an der Speicherung unserer Erinnerungen im Gedächtnis beteiligt ist.

„Das limbische System ist Teil des Gehirns aller Säugetiere. Es steuert unterschiedliche Emotionen wie Liebe, Angst oder Hass, lässt Menschen Neues lernen und ist für unser Gedächtnis zuständig '20

Im Folgenden werden die Bestandteile des limbischen Systems sowie ihre Funktionen stichwortartig dargestellt:

- Limbische Teile der Großhirnrinde (präfrontaler, orbitofrontaler und cingulärer Cortex: Bewusste Emotionen und Motive, bewusste kognitive Leistungen, Handlungs- und Impulskontrolle

- Hippocampus-Formation: Organisator des deklarativen Gedächtnisses

- Amygdala: Emotionale Konditionierung, insbesondere Vermittlung negativer Gefühle z.B. Stress und Furcht

- Mesolimbisches System: Belohnung

- Neuromodulatorische Systeme: Steuerung von Aufmerksamkeit, Motivation, Interesse, Lernfähigkeit durch die Neurotransmitter Noradrenalin, Dopamin,Serotonin und Acetylcholin. ${ }^{21}$

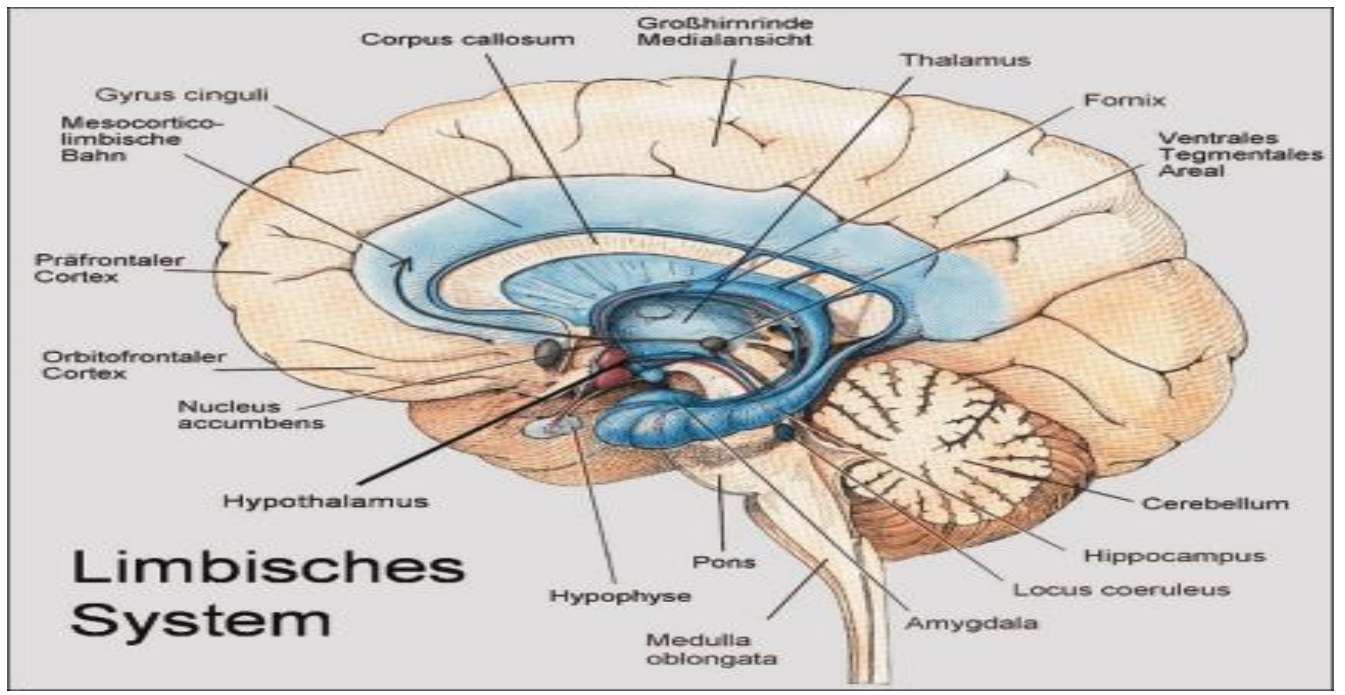

(Abbildung 27) 22

${ }^{20} \mathrm{Https://www.focus.de/wissen/mensch/limbisches-system-so-funktioniert-es} \mathrm{id} \mathrm{7124764.html,} \mathrm{am} \mathrm{1.7.2018.}$

${ }^{21}$ Vgl. Ulrich, Herrmann: 2006, S. 60. 
Das limbische System bewertet alle aufgenommenen Reize nach den Kriterien bekannt vs. unbekannt, wichtig vs. unwichtig und angenehm vs. unangenehm. In jeder Lernsituation vergleicht das limbische System den Lernstoff und die ganze Lernatmosphäre mit den bereits vorhandenen Wissensbeständen. Dabei werden ähnliche Informationen, Erfahrungen und die damit gespeicherten Emotionen aus dem Gedächtnis abgerufen. Wird der Lernstoff als wichtig, angenehm oder bekannt betrachtet, wird er gespeichert und mit den vorhandenen Netzwerken verbunden oder es werden neue Netzwerke erstellt. ${ }^{23}$

Viele moderne Gehirn- und Lernforschungen haben ergeben, dass jeder kognitive Lerninhalt zusammen mit einer Emotion bzw. einem Gefühl abgespeichert wird. Die Emotionen werden als fördernde oder hemmende-Faktoren für den Lernerfolg betrachtet.Wenn der Lernprozess von starken negativen Gefühlen geprägt ist, führt das zum Lernmisserfolg. Als Beispiel für negative Gefühle beim Lernen stehen Angst und Stress im Vordergrund.

Bei Angst oder Stress werden Adrenalin und große Menge an Noradrenalin ausgeschüttet. Blutdruck und Puls steigen, die Atmung wird verstärkt und der ganze Körper ist auf Kampf oder Flucht vorbereitet. Als Folge dazu werden die kognitiven Funktionen beeinträchtigt, jegliche Art von Kreativität wird gehemmt und das Gedächtnisvermögen lässt nach. ${ }^{24}$ Hierbei ist es wichtig, zu erwähnen, dass die richtige Menge an Noradrenalin zu positivem Stress führt, der Eustress genannt wird. Dieser Stress wird als ideal für den Lernprozess betrachtet. Die Freisetzung großer Menge an Noradrenalin führt hingegen zu negativem Stress bzw. Distress, der die Weiterleitung von Reizen unterbindet. ${ }^{25}$

Darüber hinaus haben klinische Studien festgestellt, dass negative Gefühlserfahrungen während der ersten Lebensphase, wie Verlust oder Trennung der Eltern, sich negativ auf die Aufbauprozesse der Synapsen auswirken. Diese mangelhafte Verknüpfung neuronaler Netzwerke kann später in Form von Lernstörungen erscheinen. ${ }^{26}$

22 Https://beschaffung-aktuell.industrie.de/allgemein/limbisches-system-steuert-ratio/\#slider-intro-1, am 2.1 . 2019.

${ }^{23}$ Vgl. Grein, Marion: Neurodidaktik. Grundlagen für Sprachlehrende, Hueber Verlag, Ismaning 2013, S.10.

24 Vgl. Kehrein, Anna: Strategien zum Wortschatzerwerb im Englichunterricht. Von theoretischen Betrachtungen zur Unterrichtlichen Praxis, Zentrum für Lehrerbildung Institut für Englische Philologie Didaktik des Englischen, 2013, S. 25.

${ }^{25}$ Vgl. Ulrich, Herrmann: 2006, S.24-25.

${ }^{26}$ Ebd., S. 142. 
Das heißt, dass die Kindheit und was Menschen während dieser Zeit erlebt haben, den Lernprozess positiv oder negativ beeinflussen kann. Deshalb sollte der Lehrer den psychischen Zustand des Kindes beim Lernen beobachten.

Im Gegensatz dazu fördern positive Emotionen die Lernleistung. Wenn man gut gelaunt ist und Spaß am Lernen hat, wird „Dopamin“ freigesetzt. Dopamin ist ein Neurotransmitter, der für das Weitergeben der Informationen von einem Neuron zum nächsten verantwortlich ist. Es ist hauptsächlich für Glücksgefühl, Motivation und Neugierde zuständig. Deshalb ist die Freisetzung von Dopamin beim Lernen wichtig. Es hilft auch dabei, die Wahrnehmung von Kausalzusammenhängen herzustellen und kreatives Lernen zu unterstützen. ${ }^{27}$

In Anlehnung an zahlreichen Ergebnissen der Hirnforschungen und kognitiven Untersuchungen erschienen Anfang der 1990er Jahre verschiedene Konzepte zum gehirngerichten Lehren und Lernen. Im Folgenden wird eines der weltweit verbreitesten Konzepte kurz dargestellt.

\section{Die zwölf Lehr-Lern-Prinzipien der Neurodidaktik}

Am Ende der 1980er Jahre erschien der Trend, Ergebnisse der Hirnforschungen in die Pädagogik miteinzubeziehen. Diese Idee zielte auf die Optimierung des Lernprozesses und auf die Verbesserung der Qualität des Lernens ab.

Auf zahlreichen Ergebnissen der Hirnforschung beruhend haben Renate Nummela Caine und Geoffrey Caine im Jahre 1994 zwölf Prinzipien des gehirn-fundierten Lernens und Unterrichtens (Brain-Based Lerning und Teaching) formuliert. Diese zwölf Prinzipien sollten die Lehrenden beim Unterrichten berücksichtigen, um sinnvolles Lernen verwirklichen zu können.

Diese Prinzipien sind:28

1. Lernen ist ein physiologischer Vorgang.

2. Das Gehirn ist sozial.

3. Die Suche nach Sinn ist angeboren.

4. Sinnsuche geschieht durch die Bildung von neuronalen Mustern.

5. Emotionen sind wichtig für die Musterbildung.

6. Das Gehirn verarbeitet Informationen in Teilen und als Ganzes gleichzeitig.

7. Lernen erfolgt durch gerichtete Aufmerksamkeit und durch periphere Wahrnehmung.

27 Vgl. https://blog.cognifit.com/de/was-ist-dopamin-funktionen/, am 22.7.2018.

${ }^{28}$ Vgl. Arnold, Margret: 2002, S. 107-127. 
8. Lernen geschieht sowohl bewusst als auch unbewusst.

9. Jeder Mensch besitzt verschiedene Arten, Gedächtnisinhalte zu ordnen.

10. Lernen ist entwicklungsabhängig.

11. Komplexes Lernen wird durch Herausforderungen gefördert, durch Angst und Bedrohung verhindert, was von Hilflosigkeit und Erschöpfung begleitet ist.

12. Jedes Gehirn ist einzigartig.

In Anlehnung an die Erklärung von Margret Arnold zu diesen Prinzipien, werden diese nun kurz zusammengefasst.

In Bezug auf das erste Prinzip haben moderne Hirnforschungen festgestellt, dass unser Gehirn ein lebendes System ist, wobei Körper und Geist eine vollkommene dynamische Einheit bilden. Deshalb lernt man effektiver, wenn man Erfahrungen machen kann, die die die Sinne vielfältig ansprechen.

Das zweite Prinzip bezieht sich darauf, dass das Gehirn auf Sozialverhalten hin ausgerichtet ist. Lernen ist mit der sozialen Umgebung und mit den Beziehungen zu anderen Menschen eng verbunden. Wahrnehmung, Sprache, Überzeugungen sowie Lernen sind demnach stark von zwischenmenschlichen Beziehungen beeinflusst. Deshalb lernt man effektiver, wenn soziale Interaktionen in den Lernprozess einbezogen werden.

Wie bereits erwähnt, weist das Prinzip Nummer drei darauf hin, dass das Gedächtnis nur solche Informationen, die für eine Person wichtig und von Bedeutung sind, speichert. Die Suche nach Erklärungen für Dinge und die Verbindung von Neuem mit bereits Bekanntem sind Merkmale des menschlichen Gehirns. Man kann davon ausgehen, dass Lernende effektiver lernen, wenn ihre Interessen und Erfahrungen miteinbezogen werden.

Alle Menschen ordnen ihre Umgebung mithilfe von Musterbildungen, deren Grundlage Kategorisierung von Informationen. Beim Lernen neuer Informationen wird das neue Wissen mit dem vorhandenen Vorwissen verglichen. Unterricht zielt auf die Erweiterung der Muster, die Lernende erkennen und gebrauchen, ab. Deshalb ist die Verbindung der neuen Informationen mit den bereits gespeicherten Informationen sehr wichtig für den Lernprozess.

Wie bereits gezeigt, begleiten Emotionen alle kognitiven Vorgänge und beeinflussen sie sehr. Prinzipien Nummer fünf und elf betonen, dass man effektiver lernt, wenn der Lernprozess von positiven Emotionen geprägt ist und wenn die Lernumgebung unterstützend, motivierend und herausfordernd ist.

Jedes Gehirn nimmt sowohl das Ganze als auch die Einzelteile parallel wahr. Um Informationen sinnvoll zu verarbeiten, zerlegt das Gehirn in der Tat wahrgenommene Gesamtheiten in Einzelteile. Durch die Verbindung der wahrgenommenen Details 
miteinander wird die Gesamtheit verstanden. Deshalb muss sich die Aufmerksamkeit des Gehirns sowohl auf das Ganze als auch auf Details richten.

Wenn man sich auf eine bestimmte Aufgabe konzentriert, nimmt man parallel die gesamte Umgebung, wie z.B. Hintergrundgeräusche, wahr.

Aufmerksamkeit kann durch periphere Signale leicht abgelenkt werden. Deshalb ist es wichtig, die Lernumgebung für den Lernprozess angemessen zu gestalten.

Es ist auch wichtig zu erklären, dass das Lernen in verschiedenen Schichten des Bewusstseins stattfindet. Ein Teil des Lernens und der Wahrnehmung der Informationen geschieht nicht während der Unterrichtsstunde, sondern Stunden, Tage, Wochen oder Monate später. Die Zeit, in der Informationen unbewusst weiterverarbeitet werden, nennt sich Inkubationsphase. Deshalb ist es notwendig, dass Lernende Zeit haben, ihr Lernen zu reflektieren.

Wie bereits bei der Bearbeitung des Gedächtnisses erwähnt, gibt es viele unterschiedliche Gedächtnismodelle. Diese sind: das deklarative Gedächtnis, das semantische Gedächtnis, das prozedurale Gedächtnis und das emotionale Gedächtnis. Alle diese Gedächtnisformen müssen in die Durchführung des Lernprozesses integriert werden.

Prinzip Nummer zehn betont, dass jeder Lernprozess und jede Erfahrung die Struktur der neuronalen Netzwerke im Gehirn verändern. Diese lebenslange Veränderungsbereitschaft des Gehirns nennt man Plastizität. So entwickeln sich Menschen nie genau gleich oder gleich schnell. Deshalb sollte der Lehrende die individuellen Unterschiede seiner Lernenden beim Unterrichten berücksichtigen.

Das letzte Prinzip kristallisiert heraus, dass alle Menschen über das gleiche System verfügen, sie aber trotzdem unterschiedlich sind. Die Gründe dafür sind die genetischen Unterschiede zwischen den Menschen sowie die persönliche Erfahrung und Umgebung, die bei der Entwicklung des Gehirns eine entscheidende Rolle spielen. Diese Unterschiede drücken sich in verschiedenen Lernstilen, unterschiedlichen Talenten und Intelligenzarten aus. Deshalb muss der Lehrende die Binnendifferenzierungen zwischen den Lernenden berücksichtigen und verschiedene Lern-und Denkstile in den Unterricht einbetten.

Die folgende Abbildung stellt die zwölf Prinzipien der Neurodidaktik nach Caine und Caine zusammenfassend dar: 


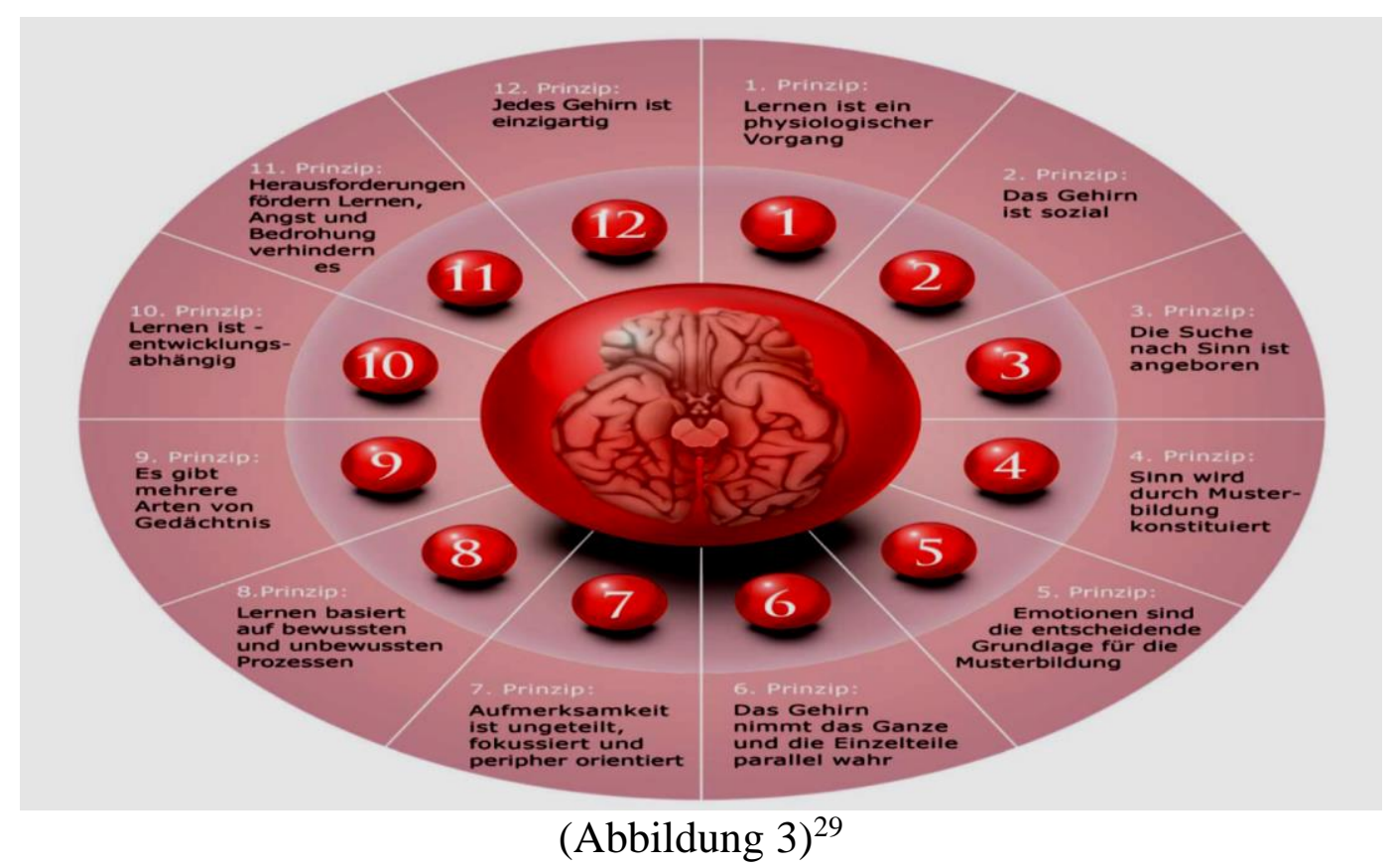

\section{Fazit}

Aus der Sicht der Hirnforschung ist das Lernen ein hochkomplexer kognitiver Vorgang, an dem alle menschliche Organe und Dispositionen beteiligt sind. Der Lernerfolg hängt also mit den Emotionen und dem emotionalen Zustand des Lernenden zusammen.

Sowohl der Lehrende als auch der Lernende müssen zwischen dem Auswendiglernen und dem sinnvollen Lernen unterscheiden. Ersteres basiert auf der Lieferung von Informationen und auf das Pauken von Fakten mit dem Ziel, Prüfung zu bestehen. Nach der Prüfung werden die gelernten Informationen unweigerlich wieder vergessen. Es geht dabei um Oberflächenwissen. Das sinnvolle Lernen hingegen basiert auf der tiefgehenden Verarbeitung von Informationen und ist mit Emotionen, Kreativität, individuellen Erfahrungen und Vorwissen verbunden. Es handelt sich um dynamisches Wissen. Der chinesische Philosoph Konfuzius hat gesagt:

", Sage es mir, und ich werde es vergessen. Zeige es mir, und ich werde es vielleicht behalten. Lass es mich tun, und ich werde es können. “30

Derzeit wird angenommen, dass der Inhalt gleichsam erfahren, erlebt und erfühlt werden soll, um fest im Gehirn verankert zu werden.

29 Del Monte, Damir: Vom Lehren, Lernen und ihrer Beforschung. SKPDW-Leitlinie, 2010, S.9 pdf.

30 Https://zitatezumnachdenken.com/konfuzius/9683, am 15.8.2018 


\section{Literaturverzeichnis}

\section{Bücherquellen}

- Arnold, Margret: Aspekte einer modernen Neurodidaktik. Emotionen und Kognitionen im Lernprozess, Verlag Ernst Vögel, München 2002.

- Auer, Christina: Fremdsprachenerwerb. Erwachsener in der Weiterbildung. Entwicklung eines teilnehmerorientierten Unterrichtkonzepts, W. Bertelsmann Verlag, Bielefeld 2013.

- Brünner, Ines: Gehirngerechtes Lernen mit digitalen Medien. Ein Unterrichtskonzept für den integrativen DaF-Unterricht, TU Berlin, Berlin 2008.

- Del Monte, Damir: Vom Lehren, Lernen und ihrer Beforschung. SKPDWLeitlinie, 2010.

- Friedrich, Gerhard: Allgemeine Didaktik und Neurodidaktik, Peter lang Verlag, Frankfurt am Main 2005.

- Grein, Marion: Neurodidaktik. Grundlagen für Sprachlehrende, Hueber Verlag, Ismaning 2013.

- Kehrein, Anna: Strategien zum Wortschatzerwerb im Englichunterricht. Von theoretischen Betrachtungen zur Unterrichtlichen Praxis, Zentrum für Lehrerbildung Institut für Englische Philologie Didaktik des Englischen, 2013.

- Roth, Gerhard: Bildung braucht Persönlichkeit. Wie Lernen gelingt, Klett-Gotta Verlag, Stuttgart 2011.

- Spitzer, Manfred: Lernen. Gehirnforschung und die Schule des Lebens, Spektrum Akad. Verlag, Heidelberg 2003.

- Ulrich, Herrmann: Neurodidaktik. Grundlagen und Vorschläge für gehirngerechtes Lehren und Lernen, Beltz Verlag, Weinheim und Basel 2006. 


\section{Internetquellen}

- https://blog.cognifit.com/de/lerntheorien/, am 4.1.2019.

- http://lexikon.stangl.eu/187/kognitivismus/, am 6.1.2019.

- https://user.phil-

fak.uniduesseldorf.de/ petersen/WiSe1516_InfowiColl/Diskurs_Konstruktivismus. pdf, am 6.1.2019.

- http://www.sciencetonic.de/200_dm_025_konzepte.html, am 6.1.2019.

- https://www.dasgehirn.info/grundlagen/anatomie/das-limbische-system, am 1.7.2018.

- https://www.focus.de/wissen/mensch/limbisches-system-so-funktioniertes_id_7124764.html, am 1.7.2018.

- https://beschaffung-aktuell.industrie.de/allgemein/limbisches-system-steuertratio/\#slider-intro-1, am 2.1. 2019.

- https://blog.cognifit.com/de/was-ist-dopamin-funktionen/, am 22.7.2018. 